\title{
THE INFLUENCE OF MECHANICAL INPUT PARAMETERS ON THE DURATION OF THE MANDIBULAR JOINT ELECTROMYOGRAPHIC SILENT PERIOD IN MAN
}

\begin{abstract}
J. O. Bailey JR., W. D. MCCall JR.* and M. M. Ash $\mathbf{J}_{\mathbf{R}}$.
Bioengineering Laboratory, Department of Occlusion, Dental Research Institute, School of Dentistry, University of Michigan, Ann Arbor, Michigan 48109. U.S.A.

Summary-The duration of the electromyographic silent period following a tap to the menton has been reported to be clinically useful in the diagnosis of mandibular joint dysfunction but not the influence of details of the tap. Using a solenoid-driven mechanical tapper, the following were investigated: in patients with joint dysfunction, effect on single muscles and position of the tap in a sequence of taps, in normal subjects, force of the tap, degree of opening of the jaw, angle of tap, and the biting force; all on the duration of the resulting silent period. No significant changes could be ascribed to any of these variables. The introduction between the teeth of wooden shims or a bite-force transducer were the only factors to change the duration. The dysfunction group had significantly longer silent periods than the normal group. It is concluded that, provided nothing is introduced between the teeth, the input parameters investigated do not influence the duration of the silent period.
\end{abstract}

\section{INTRODUCTION}

The duration of the silent period noted in electromyographic records following a tap to the menton has been advocated as a measure of mandibular jointmuscle-pain dysfunction (Bessette, Bishop and Mohl 1971; Widmalm, 1976). Since the early investigation of Brenman, Black and Coslet (1968), there have been numerous reports of EMG silent periods in both clinical (Griffin and Munro, 1971; Beemsterboer et al., 1976; Bailey, 1976) and experimental investigations (Beaudreau, Daugherty and Masland, 1969 Munro and Griffin, 1971; Sessle and Schmitt, 1972). However, there has been some question of the possible effects of different testing methods on the presence and duration, and thus the reliability, of silent period data.

Our purpose was to investigate the effect on the duration of the silent period of using various jaw muscles and varying the nature of the inducing stimulus.

\section{MATERIAL AND METHODS}

\section{Material}

Twenty persons were selected from the clinical population of the Department of Occlusion at the University of Michigan School of Dentistry. Each was questioned and examined for signs and symptoms of mandibular joint-muscle-pain dysfunction. The symptoms considered were pain in the mandibular joint. in the muscles of mastication or in both, tenderness of the muscles or joint or both upon palpation, jointsounds such as clicking or crepitation, and limitation of mandibular mobility.

To be included in the dysfunction group, two or more symptoms had to be present. To be included

* Present Address: Department of Oral Medicine School of Dentistry, State University of New York at Buffalo. Buffalo, NY 14214, U.S.A. in the normal group, a subject must have no signs or history of mandibular joint dysfunction. Ten dysfunction patients and 10 normal subjects were obtained. Ages for the normal group of subjects ranged from 21 to $56 \mathrm{yr}$ (mean 29) and for the dysfunctional group from 16 to $71 \mathrm{yr}$ (mean 27). To avoid exacerbation of the symptoms of the dysfunction patients, only a standard method, as explained below, was used to elicit silent periods. Thus the only data available from these patients were comparisons among muscles and taps in sequence.

\section{Electromyography}

Bipolar surface electrodes were used to record electrical activity from the masseter and anterior temporal muscles bilaterally and from the suprahyoid group. These signals were fed into an EMG amplifier (Grass Model 7P511) and a polygraph (Grass Model 78 ) and then to a FM tape recorder (Hewlett-Packard Model 3955) for storage at $30 \mathrm{in} / \mathrm{s}$. Upon replay at $7.5 \mathrm{in} / \mathrm{s}$ for time expansion, the duration of the silent period was measured, with Boley gauge caliper to $0.1 \mathrm{~mm}$, from the peak of the last spike preceding the inhibition to the peak of the first spike that was part of the ongoing activity. The tape speed coupled with the paper speed of $50 \mathrm{~mm} / \mathrm{s}$ led to an accuracy for silent period duration (SPD) of $0.5 \mathrm{~ms}$.

\section{Enyineering}

Bite force transducer. To investigate the influence of the bite force on SPD, a transducer to quantify biting force was required. The design parameters included an estimated maximal biting force of 200 pounds exerted in the premolar region, an insensitivity to position of force application on the transducer. registration of only the vertical force, and the smallest practical vertical dimension. The dimensions of the beam design described by Lear et al. (1965) were increased to fulfil these design parameters while keeping 
the maximum expected microstrain at less than onethird the yield point.

The calibration data were obtained with the use of foil strain gauges (Micro Measurements Model EA-90-031EC-120 Option LE) and an a.c. carrier strain-gauge amplifier (Hewlett-Packard Model 311A) with known static loads. The data were linear. The transducer was placed between the right maxillary and mandibular premolars. Four subjects also were instructed to bite at maximum, half maximum and quarter maximum force at occlusal vertical dimension while silent periods were elicited.

Mechanical tapper. To investigate the influence of the force of the menton tap on SPD, a consistent and quantifiable tap was required. A solenoid-driven mechanical tapper was designed. The shaft of the solenoid was held out by a spring and driven forcefully in when a pulse of current was applied to the coils. The system was calibrated by tapping on the bite force transducer with various amplitude current pulses.

The frame of the tapper was bolted to the back of the dental chair; a box-beam adjustment system allowed for positioning the solenoid at the menton.

Tap force calibrations. As we had collected considerable data prior to construction of the mechanical tapper, the force exerted using reflex hammer was investigated by tapping the bite-force transducer manually. The average tap force applied manually was approximately $1250 \mathrm{~g}$ force.

\section{Parameter controls}

An arbitrary standard set of parameters was established in order to investigate the influence of each parameter individually. The standard was as follows: +45 degree tap angle, contact vertical dimension, maximal clench force, a tap force of $1250 \mathrm{~g}$. Five taps were delivered to the menton at 0.5 to $1.0 \mathrm{~s}$ intervals to secure a series of five possible silent periods. Only the individual parameter deviated from this set in any one event. In the early subjects, three taps were delivered but in later subjects five taps were delivered.

To compare the-SPD among muscles, the standard elicitation as described above was used. Simultaneous silent periods were measured from each of the muscles and averaged over the five taps.

To assess the taps in the sequence, an average of the SPD was made over the four jaw closing muscles using the standard set of parameters. Comparisons were then made among taps in the sequence.

In choosing tap forces, the manual force of $1250 \mathrm{~g}$ was sclected; 625 and $1800 \mathrm{~g}$ were used also. A pilot study indicated that $2500 \mathrm{~g}$ was noxious to most subjects and that very small forces failed to elicit an observable silent pcriod. Thus these three forces included the customary manual force, a small force that was adequate to elicit the reflex and a larger force that was not noxious. These forces were delivered to the menton of the subjects by the mechanical tapper.

The effect of changing the vertical dimension was investigated by inserting wooden shims bilaterally in the posterior tooth areas. This raised the vertical dimension approximately 4,8 or $12 \mathrm{~mm}$ in the central incisor area.

Subjects were seated comfortably in a dental chair. The head support maintained the ala-tragus line hori- zontally to facilitate investigation of the effect of changing the angle of tap to the menton. Three angles, $+45,0$ and -45 degrees, relative to this alatragus line were utilized.

\section{Statistical analysis}

The results of the various tests were treated statist1cally at the 0.05 level of significance. The choice of statistical test presented a problem. As multiple measurements were made on each subject, the Friedman two-way analysis of variance ranks (Siegel, 1956) was appropriate. However, parametric tests are more powerful, so multiple paired-t tests were performed also. For these each sample was tested for normality of distribution with the Kolmogorov-Smirnov test (Siegel, 1956).

\section{RESULTS}

\section{Comparisons among muscles}

The duration of the silent periods were compared among the four jaw-closing muscles using the Friedman test (Table 1). Silent periods were not observed in the EMGs from the suprahyoid group. No significant differences were observed within the normal group $(0.3<p)$ or the dysfunction group $(0.8<p)$. The same result was obtained from these data using multiple paired- $t$ tests (all values of $p>0.1$ ).

\section{Taps in sequence}

The SPDs were compared among the taps in the sequence by averaging the durations over the four muscles used. Three taps were available from 8 normal subjects and 9 dysfunction patients. The Friedman test showed no statistically significant differences
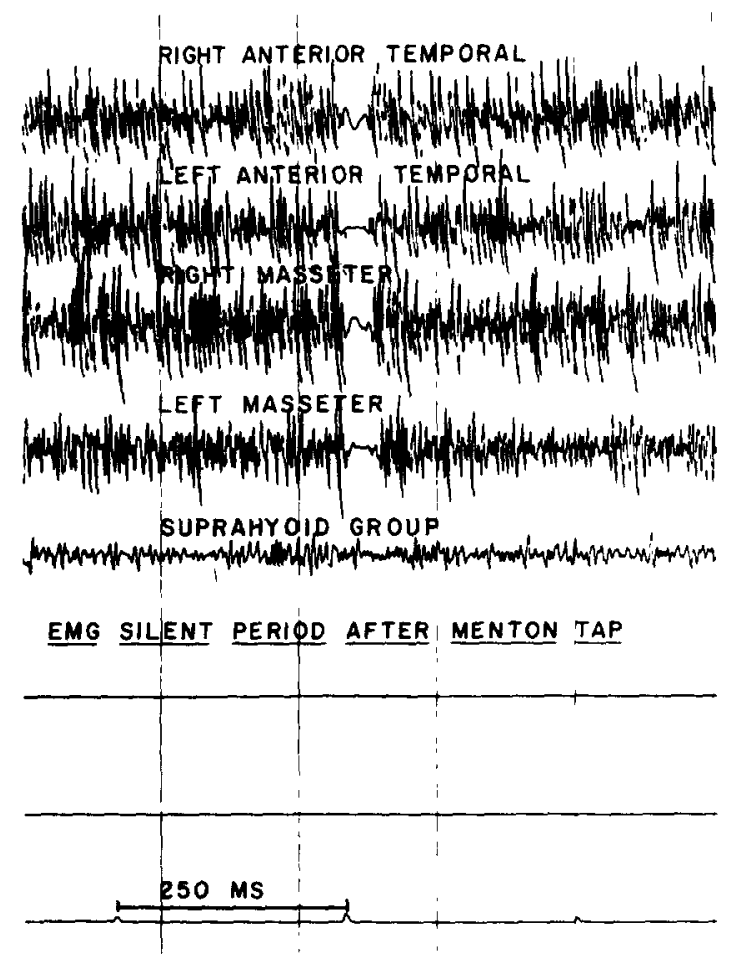

Fig. 1. EMG traces showing silent periods of like duration in elevator muscles. 
Table 1. Silent period durations (ms)

\begin{tabular}{llcccccc}
\hline \multirow{2}{*}{ Group } & \multirow{2}{*}{ Muscle } & Mean & S.D. & Min. & Max. & Conf. Interv. & N \\
\hline NORM & RAT & 26.1 & 4.1 & 21.2 & 33.3 & $22.4-29.8$ & 8 \\
NORM & LAT & 26.5 & 3.8 & 20.8 & 31.2 & $23.1-29.9$ & 8 \\
NORM & RM & 25.7 & 3.4 & 20.0 & 31.3 & $22.8-28.5$ & 9 \\
NORM & LM & 26.1 & 3.2 & 21.0 & 31.0 & $23.5-28.7$ & 9 \\
TMJ & RAT & 53.1 & 16.9 & 31.5 & 85.0 & $40.2-65.9$ & 10 \\
TMJ & LAT & 56.3 & 20.2 & 27.2 & 89.5 & $41.1-71.6$ & 10 \\
TMJ & RM & 53.9 & 19.9 & 25.7 & 89.0 & $38.9-69.0$ & 10 \\
TMJ & LM & 55.0 & 18.8 & 31.5 & 88.0 & $40.8-69.1$ & 10 \\
\hline
\end{tabular}

Rat $=$ Right anterior temporal Lat $=$ Left anterior temporal $\mathbf{R M}=$ Right masseter ; $\mathbf{L M}=$ Left masseter.

Table 2. Silent period durations (ms) five sequential taps

\begin{tabular}{lccccccc}
\hline Group & Tap & Mean & S.D. & Min. & Max. & Conf. Interv. & N \\
\hline NORM & T1 & 26.4 & 3.2 & 21.0 & 31.5 & $23.8-29.0$ & 9 \\
NORM & T2 & 26.0 & 2.8 & 20.5 & 29.5 & $23.6-28.4$ & 9 \\
NORM & T3 & 26.1 & 4.2 & 21.0 & 29.9 & $22.3-29.9$ & 8 \\
NORM & T4 & 24.9 & 2.2 & 21.2 & 27.1 & $21.8-28.0$ & 5 \\
NORM & T5 & 25.0 & 4.7 & 18.2 & 31.3 & $18.4-31.7$ & 5 \\
TMJ & T1 & 50.8 & 15.9 & 31.7 & 86.7 & $37.8-63.8$ & 9 \\
TMJ & T2 & 58.2 & 25.0 & 27.3 & 97.0 & $39.3-77.1$ & 10 \\
TMJ & T3 & 55.9 & 18.7 & 30.6 & 84.2 & $41.7-70.0$ & 10 \\
TMJ & T4 & 56.2 & 8.5 & 48.1 & 68.0 & $40.4-71.9$ & 4 \\
TMJ & T5 & 59.5 & 10.3 & 10.3 & 65.1 & - & 3 \\
\hline
\end{tabular}

among the first three taps in the sequence for either the normal group $(0.5<p)$, the dysfunction group $(0.15<p)$, or both groups combined $(0.7<p)$.

Multiple paired- $t$ tests also showed no significant differences in SPD among the taps in the sequence for either the normal (all values of $p>0.1$ ) or dysfunctional groups (all values of $p>0.1$ ). In these analyses, all five taps were included when available. (Table 2).

\section{Force of tap}

Three different force settings on the mechanical tapper were utilized to investigate the effect of tap force on the SPD in the normal subjects. Fach entry was an average value obtained over the four muscles and the sequence of taps. The mean SPD value obtained using $625 \mathrm{~g}$ was $25.8 \pm 4.4 \mathrm{~ms}$, using $1250 \mathrm{~g}$ was $27.4 \pm 9.4 \mathrm{~ms}$, and using $1800 \mathrm{~g}$ was $29.1 \pm 9.3 \mathrm{~ms}$.

The Friedman test showed no differences among the SPDs at the different tap forces $(p=0.184)$. Multiple paired- $t$ tests also showed no significant differences (all values of $p>0.1$ ).

\section{Vertical dimension}

The mean SPD values for the three vertical dimensions were $33.4,32.8,33.0 \mathrm{~ms}$. The mean SPD at each different opening was increased over that observed at contact vertical dimension. However, the mean duration at each opening did not differ from that at contact vertical dimension with statistical significance
( $0.05<p<0.10$ in each case). Further, neither the Friedman test $(0=1.00)$ nor multiple paired- $t$ tests (all values of $p<0.2$ ) showed significant differences among durations at the different openings $(4,8$ and $12 \mathrm{~mm}$ ).

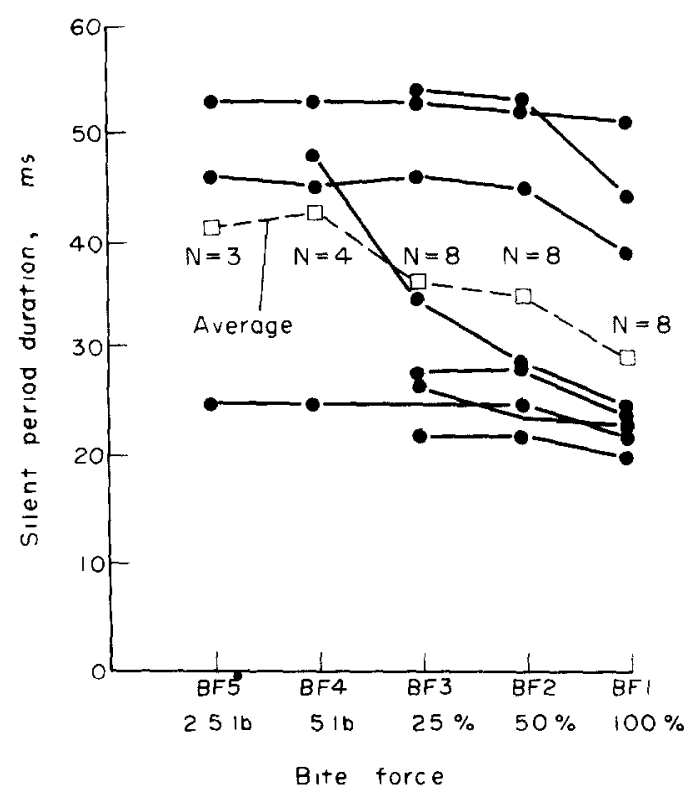

Fig. 2. Bite force effect with unilateral open vertical dimension clench. 

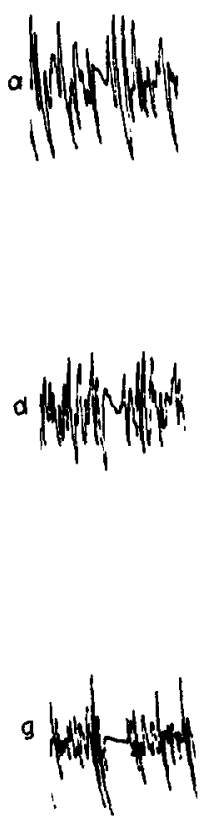
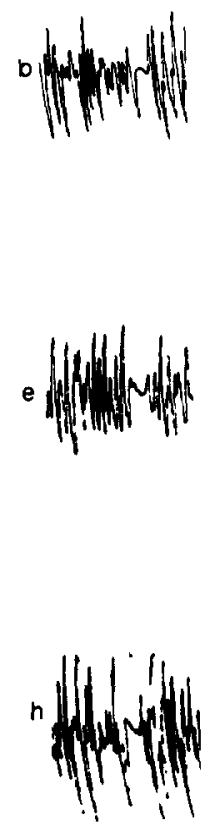
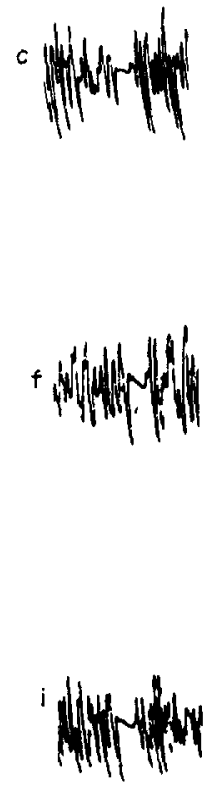

$20 \mathrm{~ms}$

Fig. 3. EMG traces of silent periods from right masseter of one subject-various representative input parameters. a. $100 \%$ b. $50 \%$ c. $25 \%$ of maximum bite force in centric occlusion. e. +45 degrees f. 0 degrees g. -45 degrees for tap angle relative to ala-tragus line. h. $+4 \mathrm{~mm} \mathrm{i.}+8 \mathrm{~mm}$.

\section{Tap angle}

Statistically significant differences in the SPDs elicited at the various angles of tap were not found (Fig. 3). The values of $p$ for the Friedman test and multiple $t$-tests were greater than 0.3 and 0.2 respectively. At 0 degrees the mean SPD was $24.8 \pm 5.1 \mathrm{~ms}$; at 45 degrees the mean SPD was $26.1 \pm 3.3 \mathrm{~ms}$; and at -45 degrees the mean was $24.1 \pm 5.0 \mathrm{~ms}$.

\section{Bite force}

Insertion of the transducer caused an increase in duration in four subjects and a decrease in four subjects when the standard circumstance was compared to the maximum bite force utilizing the transducer. Four of the eight subjects did not exhibit periods at $5 \mathrm{lb}$ bite force and five did not exhibit silent periods at $2.5 \mathrm{lb}$ bite force. The three subjects who exhibited silent periods at all force levels did not show a precipitous change in duration with change in force. The SPD while biting unilaterally on the force transducer differed from the durations elicited while the subject clenched in centric occlusion. Therefore an additional experiment testing the effect of changing bite force at occlusal vertical dimension was undertaken. The four subjects with the largest change in duration after introduction of the force transducer were utilized. These subjects were asked to clench in centric occlusion at maximal, half maximal, and quarter maximal bite force. At each force, five silent periods were elicited, averaged and compared. The group averages were $23.1,22.7$ and $24.4 \mathrm{~ms}$, respectively.

\section{Type of patient}

Statistical analyses (Mann-Whitney $U$, Median Test, and Kruskal-Wallis Test) of the differences in SPD for normal subjects versus those for individuals having joint-muscle-pain dysfunction showed a statistically significant difference between the mean values for the two groups $(p<0.001)$. Note in Tables 1 and 2 that the confidence intervals for the mean SPD never overlap.

\section{DISCUSSION}

The only study to date reporting a difference of duration among muscles is an abstract by Skiba and Laskin (1976) who were limited to one channel of EMG and thus could not compare muscles simultaneously. Further, the same method of elicitation was not used for all muscles, i.e., protrusion rather than intercuspal clench was sometimes used. We obtained no evidence that the duration was different in the four muscles. This extends the results of Bessette et al. (1971) and Widalm (1976), who compared only the masseter muscles and found no differences, and suggests that the increased duration in the dysfunction patients involves the masticatory system to a greater extent than a single side or individual muscle. If it is a single muscle problem, the influence must be pervasive and remarkably equally distributed to the motor neurons of all muscles. Our findings also suggest that the durations from only one muscle 
are necessary in an evaluation of a patient for dysfunction of the mandibular joint.

The absence of significant difference between silent period durations regardless of the position of a tap in the sequence supports the implicit assumption that the silent period is a reflex phenomenon not influenced by the inter-tap intervals used (over $0.5 \mathrm{~s}$ ).

Varying the force of the innocuous taps we used caused no change in the duration of the silent period. However, two of the subjects did not produce any silent periods with the lowest force. As the threshold may be about $625 \mathrm{~g}$, these findings suggest that the tap force should be greater than $625 \mathrm{~g}$, or at least some standard should be established. The limit of force before being noxious to the patient was between $1800-2500 \mathrm{~g}$. Thus $1250 \mathrm{~g}$ seems appropriate for eliciting an EMG silent period.

Although tap force per se does not appear to change the SPD, forces that are too small do not produce the silent period and excessive forces cause discomfort to the patient as well as double silent periods at times (Sessle and Schmitl, 1972).

In papers describing electrical stimulation (Bratzlavsky. 1972; Yu, Schmitt and Sessle, 1973), an increase in intensity of the stimulus does produce an increase in the SPD in normal subjects. This difference in the electrical versus mechanical stimulus could be due to the stimulation of different receptors.

Varying the vertical dimension, at least over the range we used, did not cause a change in SPD. The range involving 4,8 and $12 \mathrm{~mm}$ covers about onefourth the range of wide opening possible in man. There was no trend toward increased or decreased duration with increased opening, thus the experiment gave no suggestion that any change would occur at a larger jaw opening.

One aspect of a change in vertical dimension is apparent, however. The mean duration value at zero (contact vertical dimension) was different from that at 4,8 and $12 \mathrm{~mm}$. Such an increase in duration upon insertion of something between the teeth is also reported by Bessette et al. (1973). Assuming that the lack of change in silent period duration at these different vertical dimensions indicates that jaw opening plays an inconsequential role in SPD, the change in duration from contact vertical dimension to the more open dimensions could be ascribed to the different manner of contact on the maxillary and mandibular teeth in the two cases.

Our results suggest that the silent period decreases with increasing bite force. However, Bessette et al. (1973) found that the duration did not change with force variations, perhaps because they used a different method. Their subjects bit with the incisors whereas our subjects bit unilaterally in the premolar area. It may be that the instability imposed by the unilateral biting method is responsible for the different results more than the difference in bite force. This seems reasonable in light of the data showing no change of silent period duration with force changes at centric occlusion. These observations, as well as those concerning vertical dimension. seem to support the work of Goldberg (1972 his Figs. 2 and 3).

Acknowledgements This work was supported by USPHS Grant DE 02731. We thank Ms. Norma Staples for EMG assistance, Mr. E. Rohan for constructing and testing the bite force transducers. and Mr. D. Geister for designing the mechanical tapper.

\section{REFERENCES}

Bailey J. O.. Jr. 1976. The electromyographic silent period in normal subjects and patients with TMJ-muscle-pain dysfunction. M.S. thesis, University of Michigan.

Beaudreau D. E., Daugherty W. F. and Masland W. S. 1969. Two types of motor pause in masticatory muscles. Am. J. Physiol. 216, 16-21

Beemsterboer P. L., McNamara D. C., Holden S., and Ash M. M., Jr. 1977 The effect of bite plane splint on the electomyographic silent period duration. $J$. oral Rehab. In press.

Bessette R., Bishop B. and Mohl N. 1971. Duration of masseteric silent period in patients with TMJ syndrome. J. appl. Physiol. 30, 864-869.

Bessette R., Duda L., Mohl N. and Bishop B. 1973. Effect of biting force on the duration of the masseteric silent period. J. dent. Res. 52, $426-430$.

Bratzlavsky M. 1972. Pauses in activity of human jaw closing muscles. Expl Neurol. 36, 160-165.

Brenman H. S., Black M. A. and Coslet G. 1968. Interrelationship between the electromyographic silent period and dental occlusion. J. dent. Res. 47, 502 .

Goldberg L. J. 1972. The effect of jaw position on the excitability of two reflexes involving the masseter muscle in man. Archs oral Biol. 17, 565-576.

Lear C. S. C, Catz J.. Grossman R. C.. Flanagan J B. and Moorrees C. F. A. 1965. Measurement of lateral muscle forces on the dental arches. Archs oral Bıl 10 , 669-689.

Munro R. R. and Griffin C J. 1971. Electromyography of the jaw jerk recorded from the masseter and anterior temporalıs muscles in man Archs oral Biol. 16, 59-69

Ramfjord S. P. and Ash M. M.. Jr. 1971. Occlusion. 2nd Edn. Saunders. Philadelphia

Sessle B. J. and Schmitt A. 1972. Effects of controlled tooth stimulation on jaw muscle activity in man. Archs oral Biol. 17, 1597-1607.

Siegel S. 1956. Nonparametric Statistics for the Behavioral Sciences, p. 166. McGraw-Hill, New York.

Skiba T. J. and Laskın D. M. 1976. Masticatory muscle silent periods in patients with MPD syndrome. Internat Ass. for Dent. Res Preprinted abstracts, 55th Gencral Meeting, Abstract B249.

Widmalm S. E. 1976 The silent period in the masseter muscle of patients with TMJ dysfunction. Acta odont scand. 34. 43-52.

Yu S. K. J.. Schmitt A. and Sessle B. J. 1973. Inhıbitory effects on jaw muscle activity of innocuous and noxious stımulation of facial and intraoral sites in man. Archs oral Biol. 18. 861-870. 